\title{
Letter to the Editor: Comment on "Contemporary epidemiology and novel predictors of uterine rupture: a nationwide population-based study"
}

\author{
Gustavo Vilchez ${ }^{1}$ (D)
}

Received: 9 January 2018 / Accepted: 12 January 2018 / Published online: 18 January 2018

○) Springer-Verlag GmbH Germany, part of Springer Nature 2018

\section{Dear editors,}

I would like to thank Dr. Accordino and colleagues for their letter and interest in our study on contemporary epidemiology and novel predictors of uterine rupture [1]. I was pleased to read that Dr. Accordino and colleagues were able to identify the importance of the manuscript, as well as its implications on clinical practice. In spite of several changes in clinical practice along the years, as well as efforts by several organizations to decrease cesarean rates and its related morbidity; uterine rupture is still a threat [2], and previously unidentified risk factors are currently playing an important role. Therefore, it is paramount to make all the efforts and update the knowledge in this specific condition [3, 4]; particularly, after several changes that perinatal practice have undergone along the years.

A secondary purpose of their letter was to provide further information regarding the case of a patient with no apparent risk factors for uterine rupture who decided, after extensive counseling, to undergo trial of labor after cesarean delivery; in a practice with a high vaginal birth after cesarean success rate $(80 \%)$, and a low uterine rupture rate $(0.4 \%)$. Favorably, physicians were able to identify this complication promptly by ultrasonographic assessment; and therefore, prevent devastating complications in this patient.

I agree with the comments of Dr. Accordino and colleagues regarding uterine rupture. Being one of the most threatening complications in obstetrics, studies attempting to identify significant risks factors and ways to improve its prevention are warranted.
Author contributions GV: conceptualization, data curation, formal analysis, investigation, methodology, project administration, resources, software, supervision, validation, visualization, writing — original draft, writing-review \& editing.

Funding No funding received for the preparation of this manuscript.

\section{Compliance with ethical standards}

Conflict of interest The authors declare that they have no conflict of interest.

Ethical approval This article does not contain any studies with animals or humans performed by any of the authors. This letter is a response to an already published article.

Informed consent Informed consent section was not applicable for this manuscript. This letter is a response to an already published review.

\section{References}

1. Vilchez G et al (2017) Contemporary epidemiology and novel predictors of uterine rupture: a nationwide population-based study. Arch Gynecol Obstet 296(5):869-875

2. Vilchez G et al (2017) Contemporary analysis of maternal and neonatal morbidity after uterine rupture: a nationwide populationbased study. J Obstet Gynaecol Res 43(5):834-838

3. Vlemminx MW, de Lau H, Oei SG (2017) Tocogram characteristics of uterine rupture: a systematic review. Arch Gynecol Obstet 295(1):17-26

4. Shi L et al (2017) Delayed presentation of uterine rupture in a didelphys uterus misdiagnosed as appendicitis: a case report and review of the literature. Arch Gynecol Obstet 296(5):1015-1016

Gustavo Vilchez

vilchezlagosg@umkc.edu

1 Department of Obstetrics and Gynecology, University of Missouri-Kansas City School of Medicine, 2310 Holmes

St. STE 713, Kansas City, MO 64108, USA 